\title{
Three-Form Split Questionnaire Design for Panel Surveys
}

\author{
Paul M. Imbriano ${ }^{1}$ and Trivellore E. Raghunathan ${ }^{2}$
}

\begin{abstract}
Longitudinal or panel surveys are effective tools for measuring individual level changes in the outcome variables and their correlates. One drawback of these studies is dropout or nonresponse, potentially leading to biased results. One of the main reasons for dropout is the burden of repeatedly responding to long questionnaires. Advancements in survey administration methodology and multiple imputation software now make it possible for planned missing data designs to be implemented for improving the data quality through a reduction in survey length. Many papers have discussed implementing a planned missing data study using a split questionnaire design in the cross-sectional setting, but development of these designs in a longitudinal study has been limited. Using simulations and data from the Health and Retirement Study (HRS), we compare the performance of several methods for administering a split questionnaire design in the longitudinal setting. The results suggest that the optimal design depends on the data structure and estimand of interest. These factors must be taken into account when designing a longitudinal study with planned missing data.
\end{abstract}

\section{Introduction}

\subsection{Motivation for Planned Missing Data Designs}

Longitudinal of panel surveys are essential for any study of change in the key outcome variables and their correlates. As it is costly to conduct and recruit participants into such studies, researchers often try to get the most information they can out of study participants. For the purpose of design planning, survey costs may be decomposed into fixed and variable costs (Cochran 1977 chap. 5, 96-100; Groves 1989 chap. 2, 50-76). Fixed or overhead costs do not depend on sample size. In contrast, variable costs increase as the sample size of the study increases. Costs associated with sampling frame, sampling design, and questionnaire design are relatively fixed, while labor costs associated with interviewing and data entry, printing costs, and mailing costs are variable costs (Yansaneh 2005; Deutskens et al. 2006). Although lengthening a survey questionnaire will increase the cost of a study, the costs associated with adding an additional question to a questionnaire are generally small compared to the overall costs of the study (Deutskens et al. 2006; Groves 1989 chap. 10, 490-496).

Due to the costs of conducting a study, survey questions may be pooled from several investigators with multiple research interests, as it is generally cheaper to conduct a single large study instead of several small studies. This results in long questionnaires and

\footnotetext{
${ }^{1}$ Department of Biostatistics, School of Public Health, University of Michigan, 1415 Washington Heights Ann Arbor, MI 48109-2029, U.S.A. Emails: pimbri@umich.edu, and paulmimbriano@gmail.com

${ }^{2}$ Survey Research Center, Institute for Social Research, University of Michigan, 426 Thompson St, Ann Arbor, MI 48104, U.S.A. Email: teraghu@umich.edu
} 
increases the burden on participants. These studies, therefore, are also subject to dropout or nonresponse, which may lead to biased results. Sharp and Frankel (1983) found that survey length was associated with perceived burden. Furthermore, longer surveys can affect the quality of participants' responses. Several studies have shown that nonresponse rates tend to be high in surveys with long questionnaires (Adams and Darwin 1982; Dillman et al. 1993; Roszkowski and Bean 1990), and item nonresponse is more frequent towards the end of a questionnaire (Raghunathan and Grizzle 1995). Roszkowski and Bean (1990) found the response rate was $28 \%$ higher for the short version of a questionnaire compared to the long version. Past a certain length, participants become more likely to lose interest in the study, making responses less accurate (Herzog and Bachman 1981; Gonzalez and Eltinge 2007; Peytchev and Peytcheva 2017). Galesic and Bosnjak (2009) found that participants spent less time responding to items located at the end of a survey compared to items located at the beginning of the survey. Items at the end of the study also had lower response rates, shorter responses for open-ended items, and less variation in responses when items on the same response scale were arranged in a grid. A shorter survey length alleviates these problems and potentially decreases the cost of data collection per subject. The goal, therefore, is to balance collecting a set of rich variables while not placing an undue burden on participants.

The problems caused by lengthy questionnaires are exacerbated in longitudinal studies due to calling on respondents to fill out the questionnaires repeatedly. In addition to the issues of fatigue and bias from a lengthy survey, longitudinal studies must also take into consideration the effect that a lengthy survey may have on attrition. Sharp and Frankel (1983) found that, when asked if they would agree to participate in a second interview one year later, a higher proportion of participants who received the longer survey $(27 \%)$ indicated that they would not agree to a follow-up interview compared to the group that received the shorter survey (13\%). The actual follow-up rates one year later were closer for the long versus short survey groups ( $85 \%$ versus $88 \%$ ), but still favored the shorter interview group. Zabel (1998) found that a planned reduction in the length of interviews for the Panel Study of Income Dynamics led to a decrease in the attrition rate.

\subsection{Planned Missing Data Designs and Split Questionnaires}

Meanwhile, advancements in software for handling missing data, especially the multiple imputation approach, have made missing data less problematic for data analysis, and, in fact, designing a survey to purposely include missing data could improve the quality of the study (Littvay 2009). A planned missing data design provides an effective way to reduce questionnaire length, while maintaining all relevant questions of interest. Furthermore, missing values resulting from the planned missing approach are by design either missing completely at random (MCAR) or missing at random (MAR) (Rubin 1976; Little and Rubin 2002 chap. 1, 11-13). As a result, we can use multiple imputation, maximum likelihood, or fully Bayesian approaches to handle the missing data just by focusing on the model for variables in the survey. In fact, since planned missing data designs reduce the burden on participants, the probability of nonresponse decreases, making it less likely to observe values that are missing not at random (MNAR), and, as a result, multiple imputation and maximum likelihood approaches are more likely to be valid (Rhemtulla 
and Little 2012; Jorgensen et al. 2014; Kaplan and Su 2016). Planned missing data approaches have frequently been used for educational assessment, where students are evaluated on several subjects. Evaluating a student's proficiency on every subject would take a great deal of time, making it disruptive for students and unlikely to be approved by administrators. For this reason, many assessments utilize either split questionnaire design or multiple matrix sampling, where each student responds to just a subset of the total questions. The Kentucky Instructional Results Information System, the Massachusetts Comprehensive Assessment System, the National Assessment of Educational Progress, and the Dutch National Assessment Program have all used multiple matrix sampling to reduce the testing burden on students (Childs and Jaciw 2002).

Raghunathan and Grizzle (1995) proposed split questionnaire design as an extension of multiple matrix sampling described in Shoemaker (1973), which randomly sampled items for each individual. Raghunathan and Grizzle (1995) modified multiple matrix sampling to place constraints on item assignment so that all population quantities of interest were estimable. Split questionnaire design divides the survey questions into multiple components and each participant responds to a fraction of the total components. One common variant, the three-form split questionnaire, divides the survey into four components (X,A,B,C). Each participant responds to all items in $\mathrm{X}$ and two of the three other components, resulting in three unique survey forms, $(\mathrm{X}, \mathrm{A}, \mathrm{B}),(\mathrm{X}, \mathrm{A}, \mathrm{C})$ and $(\mathrm{X}, \mathrm{B}, \mathrm{C})$, which are administered in equal proportions (Graham et al. 2006; Rhemtulla and Little 2012). This particular three-form design reduces the survey length by approximately $25 \%$, but modifications can be made to both the number of total components used and the fraction that each participant answers, depending on the survey composition and desired reduction in length. The split questionnaire is simple to implement and has been used in multiple studies (Graham et al. 2006).

\subsection{Considerations for Implementing Split Questionnaire Designs}

It is important to note that the ordering of questions within a questionnaire and where a question appears in relation to other questions could have an impact on responses (Schuman and Presser 1981; Sudman et al. 1996). Context effects should be considered during the design phase. Some context effects can be accounted for by placing variables into blocks. The questions within each block can be specifically ordered to account for context effects and the blocks themselves divided into components instead of individual variables. This should be done if certain questions are known to impact the answers to other questions. However, we might not always know if the ordering of questions will affect responses. Thus, when we design a split questionnaire, omitting certain questions or altering the ordering of questions could alter responses and possibly lead to bias. For split questionnaire designs, it would be useful to check that there are no systematic differences in responses to questions based on the assigned survey portion.

In cross-sectional studies, the split questionnaire design was found to produce estimates similar to those obtained in the absence of missing data (Raghunathan and Grizzle 1995; Littvay 2009), but with a decrease in power. The loss of statistical power can be somewhat mitigated by the increased sample size obtainable due to the decrease in cost per participant (Thomas et al. 2006; Littvay 2009). This is especially true if certain individual 
items are expensive to measure. Peytchev and Peytcheva (2017) also demonstrated that responses to questions from a split questionnaire design more closely resembled responses when questions are at the beginning of a lengthy survey instead of at the end, indicating that a split questionnaire may decrease bias due to fatigue. The application of a split questionnaire design should be considered when it would decrease the mean squared error (MSE) compared to a traditional survey. Unfortunately, it is difficult to know when a split questionnaire design would decrease the MSE, as the amount of bias due to burden from survey length will likely differ among studies. Although Sharp and Frankel (1983) demonstrated that an increase in survey length led to an increase in perceived burden, both the perceived usefulness of the survey and invasiveness of the questions had a larger effect on burden. A split questionnaire design could have a larger impact on the MSE for longitudinal studies if it substantially lowers the dropout rate. Fewer dropouts might decrease the variance, through an increased sample size at later study visits, and decrease nonresponse bias compared to a traditional longitudinal study.

\subsection{Literature Review of Longitudinal Split Questionnaire Design}

Until recently, little research had been done on the implementation of split questionnaires for longitudinal studies (Jorgensen et al. 2014). Creech et al. (2011) found that groups administered a split questionnaire had lower attrition than the groups administered the full questionnaire. Gonzalez and Eltinge (2008) and Gonzalez (2012) examined adaptively assigning items to individuals for the second interview in a panel study based on responses from the first interview. Rhemtulla and Little (2012) mentioned three strategies for assigning forms in longitudinal studies on growth curves: assigning the same forms at each visit, rotating the forms, or randomly assigning forms at each wave. It was suggested that the same forms should be given at each visit when the measure of a variable across waves is of primary interest and the forms should be rotated when examining the relationship of several latent variables. Jorgensen et al. (2014) examined longitudinal three-form split questionnaire designs in a latent variable setting. They explored the effect of longitudinal form assignment on the relative efficiency of estimating cross-lagged regression parameters (the association of a variable with another variable measured at an earlier time), autoregression parameters (the association of a variable with itself measured at different times), and factor loadings. They implemented three longitudinal form designs: assigning the same form at each visit, assigning a different form at each visit, and randomly assigning forms at each visit. In general, the same form was better at estimating cross-lagged regression and autoregression parameters, but different forms were better for factor loading. The random form assignment performed somewhere in between the same form and different form designs.

In Section 2, we propose several longitudinal designs for split questionnaires. In addition to the three assignment methods examined in Jorgensen et al. (2014), we explore several more complex methods to determine whether the more complex methods provide additional benefits in estimation compared to simpler assignment methods. In Section 3, we discuss methods to analyze data collected from a split questionnaire survey. We compare the performance of our proposed split questionnaire designs using results from simulations in Section 4 and data from the Health and Retirement Study (HRS) in Section 5. Finally, we discuss our conclusions, limitations, and areas for future research in Section 6. 


\section{Longitudinal Split Questionnaire Survey Designs}

\subsection{Issues in Longitudinal Split Questionnaire Design}

Administering split questionnaires in longitudinal surveys is more complex due to repeated variable measurements on the same subject at different time points. Usually, these repeated measurements are highly correlated (Hardt et al. 2012). Also, unlike crosssectional studies, not all variables are collected at once. A longitudinal study where participants skip certain study visits could be considered for a longitudinal split questionnaire, if the cross-sectional study questionnaire is not too burdensome. However, when the cross-sectional questionnaire is lengthy, it is more sensible for a longitudinal split questionnaire study to be comprised of cross-sectional split questionnaires administered at each visit. We might consider designing a new split questionnaire at each wave conditional on the variables already observed in the previous waves (here we define wave as any study visit in which survey questionnaires were administered to participants). However, in most studies there will be a large number of possible split questionnaire designs to choose from. Assuming we have an equal number of variable blocks in each split, there are

$$
\frac{\left(\begin{array}{l}
b \\
q
\end{array}\right)\left(\begin{array}{c}
b-q \\
q
\end{array}\right) \ldots\left(\begin{array}{l}
2 q \\
q
\end{array}\right)\left(\begin{array}{l}
q \\
q
\end{array}\right)}{s !}
$$

number of potential split questionnaire designs. Here $b$ represents the total number of variable blocks, where each block consists of one or more variables. Certain variables may be placed in the same block based on content and skip pattern. For example, a question asking, "Do you currently smoke?" may be placed in the same block as a question related to, "How many cigarettes do you smoke a day?" In the equation, s represents the number of splits or survey components into which the variable blocks are divided. Finally, q denotes the number of blocks per split. Here, we are assuming that the order of the splits does not matter. This is a reasonable assumption if there are no context effects, which have hopefully been accounted for when placing variables into blocks. With eighteen blocks and three splits, there are 2,858,856 potential split questionnaire designs to choose from. There are even more possible designs to consider if the order of splits affects responses. This can make it difficult to design a new split questionnaire at every visit. It would be simpler instead to use the same cross-sectional split questionnaire forms at each wave. Although the same forms are being used, each individual does not need to receive the same form at every visit. There are still multiple ways that we can administer the forms in a longitudinal design. We propose several methods for administering a three-form split questionnaire design in longitudinal studies.

Prior to designing a study, we need to determine whether to administer the same form to each participant throughout the entire study, rotate the form each participant receives from visit to visit, or employ some combination of those two designs, in which we administer the same form to some participants and rotate the forms for other participants. Which design works best likely depends on the correlation structure of the data, more specifically, how the correlation between components measured at the same wave (within-wave 
correlation) compares to the correlation of measurements on the same variable over time (autocorrelation) and the correlation between two separate components measured at different waves (between-wave correlation). When the autocorrelation is greater than the within-wave correlation, we expect it would be preferable for most estimates to not measure the same components at each wave, as the missing values would be highly correlated with the variable observed at other waves. We expect that this would improve estimation (Raghunathan and Grizzle 1995; Thomas et al. 2006). Note that in this article, we use the term autocorrelation to refer to a variable's correlation with itself over time. This should not be confused with the use of the term autocorrelation in the context of time series analysis, where autocorrelation refers to the correlation of residuals from a regression model across time points (Box and Pierce 1970). We also hypothesize that when most correlations are non-zero, more complex form rotations will produce more precise estimates by better estimating these different correlations. We will examine this hypothesis in our analysis and simulations. Which quantity is of primary interest to investigators could also influence which design should be administered. If we are primarily interested in estimating the change in a variable over time, it might be preferable to administer the same components throughout the study so that a variable can be measured in the same individuals at all time points.

\subsection{Proposed Longitudinal Split Questionnaire Designs}

We consider six different design options for allocating a three-form questionnaire in longitudinal studies. Table 1 displays how each component, A, B, and C, would be allocated under the first five proposed designs in a three-wave study, but each design could be easily modified depending on the number of waves and desired reduction in survey length. For the first two options, the participants are placed into three groups. With Option 1 , each group receives either form $(A, B),(A, C)$, or $(B, C)$ throughout the study, while for Option 2 the forms each group receives are rotated in a manner that allows each form to be given in equal proportions at each wave. Option 1 is analogous to the same form design in Jorgensen et al. (2014). We expect it to perform better at estimating the change in a variable over time as it performed better at estimating autoregression parameters in Jorgensen et al. (2014). Option 2 is analogous to the different form design in Jorgensen et al. (2014). For Option 3, we again administer to participants a different form at each wave, but we instead create six groups, which provides more ways of cycling the forms. We hypothesize that the more complex design of Option 3 may allow us to better model variable correlations than Option 2 and will outperform it when most correlations are fairly strong. We will examine this hypothesis through our numerical analysis and simulations.

Combining aspects of the first two options may produce split questionnaire designs that perform well in estimating both the cross-sectional and longitudinal properties of variables. We would expect Option 1 to better estimate longitudinal properties, such as the change in a variable over time, as the same variables in individuals are measured at all waves. However, when autocorrelations are large, we expect that rotating the forms would perform better at estimating cross-sectional properties, such as the variable means at each wave, than administering the same form at each wave because a variable is likely more predictive of its missing value than other variables. Collecting variables that are highly 
Table 1. Form allocation by design option.

\begin{tabular}{|c|c|c|c|}
\hline Option & Wave 1 & Wave 2 & Wave 3 \\
\hline \multirow{3}{*}{1} & $\mathrm{AB}$ & $\mathrm{AB}$ & $\mathrm{AB}$ \\
\hline & $\mathrm{AC}$ & $\mathrm{AC}$ & $\mathrm{AC}$ \\
\hline & $\mathrm{BC}$ & $\mathrm{BC}$ & $\mathrm{BC}$ \\
\hline \multirow{3}{*}{2} & $\mathrm{AB}$ & $\mathrm{AC}$ & $\mathrm{BC}$ \\
\hline & $\mathrm{AC}$ & $\mathrm{BC}$ & $\mathrm{AB}$ \\
\hline & $\mathrm{BC}$ & $\mathrm{AB}$ & $\mathrm{AC}$ \\
\hline \multirow{6}{*}{3} & $\mathrm{AB}$ & $\mathrm{AC}$ & $\mathrm{BC}$ \\
\hline & $\mathrm{AB}$ & $\mathrm{BC}$ & $\mathrm{AC}$ \\
\hline & $\mathrm{AC}$ & $\mathrm{BC}$ & $\mathrm{AB}$ \\
\hline & $\mathrm{AC}$ & $\mathrm{AB}$ & $\mathrm{BC}$ \\
\hline & $\mathrm{BC}$ & $\mathrm{AB}$ & $\mathrm{AC}$ \\
\hline & $\mathrm{BC}$ & $\mathrm{AC}$ & $\mathrm{AB}$ \\
\hline \multirow{6}{*}{4} & $\mathrm{AB}$ & $\mathrm{AB}$ & $\mathrm{AB}$ \\
\hline & $\mathrm{AB}$ & $\mathrm{AC}$ & $\mathrm{BC}$ \\
\hline & $\mathrm{AC}$ & $\mathrm{AC}$ & $\mathrm{AC}$ \\
\hline & $\mathrm{AC}$ & $\mathrm{BC}$ & $\mathrm{AB}$ \\
\hline & $\mathrm{BC}$ & $\mathrm{BC}$ & $\mathrm{BC}$ \\
\hline & $\mathrm{BC}$ & $\mathrm{AB}$ & $\mathrm{AC}$ \\
\hline \multirow{9}{*}{5} & $\mathrm{AB}$ & $\mathrm{AB}$ & $\mathrm{AB}$ \\
\hline & $\mathrm{AB}$ & $\mathrm{AC}$ & $\mathrm{BC}$ \\
\hline & $\mathrm{AB}$ & $\mathrm{BC}$ & $\mathrm{AC}$ \\
\hline & $\mathrm{AC}$ & $\mathrm{AC}$ & $\mathrm{AC}$ \\
\hline & $\mathrm{AC}$ & $\mathrm{BC}$ & $\mathrm{AB}$ \\
\hline & $\mathrm{AC}$ & $\mathrm{AB}$ & $\mathrm{BC}$ \\
\hline & $\mathrm{BC}$ & $\mathrm{BC}$ & $\mathrm{BC}$ \\
\hline & $\mathrm{BC}$ & $\mathrm{AB}$ & $\mathrm{AC}$ \\
\hline & $\mathrm{BC}$ & $\mathrm{AC}$ & $\mathrm{AB}$ \\
\hline
\end{tabular}

predictive of missing values is helpful for multiple imputation (Collins et al. 2001; Thomas et al. 2006; Hardt et al. 2012). There may also be scenarios where one of Option 1 or Option 2 does not perform well, but a design combining aspects of the two options still performs fairly well. Option 4 represents a combination of the first and second options. For this option, there are a total of six groups and half of the participants receive the same form throughout and the other half follow the rotation for Option 2. Option 5, the most complex of the planned study designs, contains nine groups, three of which receive the same forms as in Option 1 and the other six are rotated like in Option 3.

The final design, Option 6, randomly assigns forms at each wave, making it simple to administer. This option allows every possible rotation to occur, which we believe is beneficial when most correlations are non-zero, but makes the form design unbalanced. For example, under Option 6, we no longer control the number of individuals who receive form AC after receiving AB. Random form assignment was also examined in Jorgensen et al. (2014), where it was found to perform somewhere in between the same and different form designs. Our hypothesis is that the more complex designs would perform well under 
many types of correlation structures and for multiple estimands, but might not necessary be optimal under any scenario or for any estimate. Depending on the performance of proposed design options and estimands of interest, this may be a worthwhile trade-off.

\section{Analysis of Split Questionnaire Surveys}

Due to the presence of missing data, maximum likelihood, fully Bayesian approaches, or multiple imputation are necessary for data analysis. We focus on results from data analysis using both maximum likelihood and multiple imputation for comparing the performance of our proposed split questionnaire designs.

\subsection{Maximum Likelihood Estimation}

Missing values resulting directly from the split questionnaire survey implementation are by design. Because of this, these missing values are either MCAR or MAR. Therefore, we can ignore the missing data mechanism and base our inference on only the observed data likelihood. For most instances, we can compute the maximum likelihood estimator (MLE) using an iterative method, such as Newton-Raphson or the EM algorithm and asymptotic standard errors can be obtained by inverting the Fisher information matrix or using other methods like bootstrapping (Little and Rubin 2002 chap. 9, 190-199).

In the case where complete data from each individual follows a $p$ dimensional multivariate normal distribution with mean $\mu$ and variance $\Sigma$, we can obtain closed form solutions for the information matrix. Hartley and Hocking (1971) demonstrated how to compute the Fisher information of the joint likelihood of $\mathrm{Y}_{1}, \ldots \mathrm{Y}_{\mathrm{n}}$ for any arbitrary missing data pattern.

For each observation, $\mathrm{Y}_{\mathrm{i}}$, we can construct a $p \times p$ matrix such that for $1 \leq j \leq p$ each entry $a_{j, j}=1$ if variable $j$ was observed and 0 otherwise. We can then take $d_{i}$ equal to this constructed matrix after all rows of zeros have been deleted, creating a $o_{i} \times p$ matrix where $o_{i}$ denotes the number of variables observed on subject $i$. Each observation $\mathrm{Y}_{\mathrm{i}}$ follows a multivariate normal distribution with mean $d_{i} \mu$ and variance $d_{i} \Sigma d_{i}^{T}$.

The expected information matrix is block diagonal in the multivariate normal case, allowing us to compute and invert the expected information for $\mu$ and $\Sigma$ separately. For $\mu$ we can conveniently write the expected Fisher information for the total sample as

$$
I_{\mu}=\sum_{i=1}^{n} \mathrm{~d}_{\mathrm{i}}^{\mathrm{T}}\left(\mathrm{d}_{\mathrm{i}} \Sigma \mathrm{d}_{\mathrm{i}}^{\mathrm{T}}\right)^{-1} \mathrm{~d}_{\mathrm{i}}
$$

(Hartley and Hocking 1971). We can obtain the asymptotic variance of $\mu$ by inverting $\mathrm{I}_{\mu}$, assuming that $\Sigma$ is either known or we have a consistent estimate of $\Sigma$. The variance obtained from inverting this matrix is asymptotically equivalent to the variance from using the best linear unbiased estimation (BLUE) method described by Chipperfield and Steel (2009).

Now, let $\sigma_{j k}$ denote the element of $\Sigma$ located at row $j$ and column $k$. For the information matrix of $\Sigma$, it is convenient to create a vector of length $\left({ }_{2}^{p+1}\right), \delta$, which contains all unique elements of $\Sigma$, or all $\sigma_{j k}$ such that $j \leq k$. The information matrix based on $\delta$ is a $\left({ }_{2}^{p+1}\right) \times\left({ }_{2}^{p+1}\right)$ matrix. Let $\Sigma_{\mathrm{i}}=\mathrm{d}_{\mathrm{i}} \sum \mathrm{d}_{\mathrm{i}}^{\mathrm{T}}$ denote the variance matrix for subject $i$ and let $\mathrm{I}_{\Sigma \mathrm{i}}$ denote the 
expected information from subject $i$. The formula for the expected information for subject $i$, corresponding to the negative expectation of the partial derivative, is as follows:

$$
-E\left[\frac{\partial^{2} \log L}{\partial \sigma_{j k} \partial \sigma_{l m}}\right]=\frac{1}{2} \operatorname{tr}\left(\Sigma_{\mathrm{i}}^{-1} \Sigma_{\mathrm{ijk}} \Sigma_{\mathrm{i}}^{-1} \Sigma_{\mathrm{ilm}}\right),
$$

where $\Sigma_{\mathrm{ijk}}$ is a $o_{i} \times o_{\mathrm{i}}$ matrix with entries corresponding to elements $\sigma_{j k}$ and $\sigma_{k j}$ in $\Sigma_{\mathrm{i}}$ equal to one and all other elements equal zero (Hartley and Hocking 1971). Thus, $-E\left[\frac{\partial^{2} \log L}{\partial \sigma_{j k} \partial \sigma_{l m}}\right]=0$ if $\sigma_{j k}$ or $\sigma_{i m}$ are not elements of $\Sigma_{i}$. From this formula we can calculate the expected information matrix for each observation. Due to the additive property of Fisher information, the total expected information for $Y_{1}, \ldots Y_{n}$ is equal to the sum of the expected information for each individual observation. Therefore,

$$
\mathrm{I}_{\Sigma}=\sum_{i=1}^{n} \mathrm{I}_{\Sigma \mathrm{i}}
$$

where $I_{\Sigma}$ is the expected Fisher information for $\Sigma$. We can obtain the asymptotic variances for our MLE estimates of $\mu$ and $\Sigma$ by inverting the expected information matrices, assuming that all elements of $\mu$ and $\Sigma$ are estimable from our study. We can use the variances obtained from the expected information matrix to directly compare the efficiency of two proposed split questionnaire designs in estimating certain quantities. For the multivariate normal distribution, the expected information matrix does not depend on $\mu$. We can directly compute the expected information matrix if $\Sigma$ is known, which enables a comparison of two proposed split questionnaire designs when the covariance structure of the data is known. When $\Sigma$ is unknown, we can iteratively solve for $\mu$ and $\Sigma$, as described by Hartley and Hocking (1971), and obtain estimates for the large-sample variance-covariance matrices of our parameter estimates based on the expected Fisher information. It is suggested that estimating the variance based on the observed information is generally preferable in the presence of missing data, as the observed information provides valid estimates for the asymptotic variance when data are MAR, while the expected Fisher information requires that the missing data mechanism is correctly specified in order to produce consistent estimates of the variance (Kenward and Molenberghs 1998; Little and Rubin 2002, chap. 11, 223-227). The expectation of the observed information should be taken with respect to the joint distribution of the data and the missing data mechanism. Ignoring the missing data mechanism when taking the expectation (as we have done here) is only valid when data are MCAR. However, for the purpose of comparing our proposed split questionnaire designs, missing data are MCAR by design.

\subsection{Multiple Imputation}

Multiple imputation (MI) is a frequently used method for handling missing data (Chhabra et al. 2017). With MI, we fill in missing values to create several complete data sets. The MI approach is particularly convenient because, after performing MI, standard statistical analysis can be applied on each imputed data set. The estimated parameters and variances from each analysis are combined using Rubin's rules (Rubin 1987, 76) to obtain our final estimates and variances. Often, multiple imputation is done using a Sequential Regression Multiple Imputation (SRMI) framework, where missing values are drawn using Gibbs 
sampling from the posterior predictive distribution of a regression model, with each missing variable regressed on all other variables (Raghunathan et al. 2001). Several popular multiple imputation software packages, like IVEWare and MICE, use the SRMI framework (Raghunathan et al. 2002; Van Buuren and Groothuis-Oudshoorn 2011).

Since imputation uses information from other variables to estimate missing values, having observed values highly correlated with missing values will better predict those missing values and improve imputations (Collins et al. 2001; Thomas et al. 2006; Hardt et al. 2012). Ideally, we would design split questionnaire surveys that take advantage of this attribute; for this reason, Raghunathan and Grizzle (1995) assign variables using correlations, where variables with high partial correlations are placed in different components. For most cross-sectional studies, we simply administer forms $\mathrm{AB}(\mathrm{X}, \mathrm{A}, \mathrm{B})$, $\mathrm{AC}(\mathrm{X}, \mathrm{A}, \mathrm{C})$, and $\mathrm{BC}(\mathrm{X}, \mathrm{B}, \mathrm{C})$ in equal proportions and focus our attention on how to place the variables into each component.

\section{Simulation and Analysis with Proposed Split Questionnaire Designs}

\subsection{Comparing the Performance of Split Questionnaire Designs}

We performed simulations to examine how well the proposed designs perform under a number of different correlation structures when data follow a multivariate distribution. Several papers describe methods for determining optimal split questionnaire designs. Thomas al. (2006) focused on assigning split questionnaires so observed values would be more predictive of missing values. Chipperfield and Steel (2009) considered maximizing the efficiency of estimated population totals for a fixed cost or minimizing the cost for a fixed variance. Chipperfield and Steel (2011) examined optimal split questionnaire designs with respect to costs for estimating either variable means for multivariate normal data, probabilities for multinomial data, or parameter estimates for linear regression. Chipperfield et al. (2018) examined split questionnaire designs for binary variables in terms of minimizing the loss of information for a generalized logistic regression model. Here, there is a single outcome and multiple covariates. Adiguzel and Wedel (2008) proposed minimizing the Kullback-Leibler (KL) divergence between the observed and complete data likelihoods for determining an optimal split questionnaire design. The KL divergence is a measurement of the distance between two probability distributions. The idea is to find the split questionnaire design that will produce an observed data likelihood that is closest to the complete data likelihood in the absence of missing data. All of these papers looked at optimal designs for cross-sectional studies. For the purpose of longitudinal designs, we want to include estimation of change in a variable over time. We also want to consider that there may be several regression models fit to the data and do not want to distinguish between outcome variables and covariates. Also, depending on the purpose of the study, certain estimands may be of more interest than others and this may not be captured by a single overall summary measure like KL divergence.

We examined the performance of our proposed design options in terms of how well they estimated three key quantities of interest: variable means, variance-covariance components, and the linear change in variable means over time. The estimates for the means and variance-covariance provide all the information necessary to characterize the 
multivariate normal distribution. For most surveys, investigators are primarily interested in estimating the population mean of a variable or variables, or in performing regression analysis. For multivariate normal data, estimated parameters from a non-repeated linear regression model can be obtained by re-parameterizing the mean and variance-covariance estimates. The design that better estimates these parameters will produce better regression estimates. One of the major reasons for conducting a longitudinal study is to assess change in a variable over time (Cook and Ware 1983). Hence, the change over time is likely of interest to investigators. We estimated our quantities of interest using both maximum likelihood estimation and multiple imputation.

\subsection{Simulation Setup}

For our data, we took three variables at each wave to represent the A, B, and C components of the split questionnaire with the $\mathrm{X}$ component omitted for simplicity, using a sample size of 108. The nine variables come from a multivariate normal distribution with the variancecovariance structure shown in Table 2 . We used five parameters for the covariances, $\rho_{1}$ denoting the within-wave correlation, $\rho_{2}$ and $\rho_{3}$ the autocorrelations, and $\rho_{4}$ and $\rho_{5}$ representing the correlation between two separate components measured at different waves. The means of each variable changed linearly over time.

By varying the values of $\rho_{1}, \rho_{2}, \rho_{3}, \rho_{4}$, and $\rho_{5}$ we can create numerous correlation structures. Table 3. displays the correlations we used to test the performance of different options. For the first three correlation structures, only one of the within-wave, autocorrelation, or between-wave correlation is non-zero, enabling easy comparisons between the performance of each design under extremely different conditions. These first three correlation structures are not intended to represent typical correlation structures for repeated measures data, though we may find examples of variables that follow a similar correlation structure to Structure 1 and Structure 2. Say one of the variables represents a binary indicator of an infectious disease (like the flu) and the other variables represent possible symptoms related to that disease (such as fever). If enough time passes between consecutive longitudinal waves such that any acute infection will likely have resolved, we might observe a correlation similar to Structure 1. In this instance, we would expect

Table 2. Variance-covariance structure.

\begin{tabular}{ll|ccc|ccc|ccc}
\hline & & \multicolumn{3}{|c|}{ Wave 1 } & \multicolumn{3}{c|}{ Wave 2 } & \multicolumn{3}{c}{ Wave 3 } \\
& & $\mathrm{A}$ & $\mathrm{B}$ & $\mathrm{C}$ & $\mathrm{A}$ & $\mathrm{B}$ & $\mathrm{C}$ & $\mathrm{A}$ & $\mathrm{B}$ & $\mathrm{C}$ \\
\hline \multirow{4}{*}{ Wave 1 } & $\mathrm{A}$ & 1 & $\rho_{1}$ & $\rho_{1}$ & $\rho_{2}$ & $\rho_{4}$ & $\rho_{4}$ & $\rho_{3}$ & $\rho_{5}$ & $\rho_{5}$ \\
& $\mathrm{~B}$ & $\rho_{1}$ & 1 & $\rho_{1}$ & $\rho_{4}$ & $\rho_{2}$ & $\rho_{4}$ & $\rho_{5}$ & $\rho_{3}$ & $\rho_{5}$ \\
& $\mathrm{C}$ & $\rho_{1}$ & $\rho_{1}$ & 1 & $\rho_{4}$ & $\rho_{4}$ & $\rho_{2}$ & $\rho_{5}$ & $\rho_{5}$ & $\rho_{3}$ \\
\hline \multirow{4}{*}{ Wave 2 } & $\mathrm{A}$ & $\rho_{2}$ & $\rho_{4}$ & $\rho_{4}$ & 1 & $\rho_{1}$ & $\rho_{1}$ & $\rho_{2}$ & $\rho_{4}$ & $\rho_{4}$ \\
& $\mathrm{~B}$ & $\rho_{4}$ & $\rho_{2}$ & $\rho_{4}$ & $\rho_{1}$ & 1 & $\rho_{1}$ & $\rho_{4}$ & $\rho_{2}$ & $\rho_{4}$ \\
& $\mathrm{C}$ & $\rho_{4}$ & $\rho_{4}$ & $\rho_{2}$ & $\rho_{1}$ & $\rho_{1}$ & 1 & $\rho_{4}$ & $\rho_{4}$ & $\rho_{2}$ \\
Wave 3 & $\mathrm{A}$ & $\rho_{3}$ & $\rho_{5}$ & $\rho_{5}$ & $\rho_{2}$ & $\rho_{4}$ & $\rho_{4}$ & 1 & $\rho_{1}$ & $\rho_{1}$ \\
& $\mathrm{~B}$ & $\rho_{5}$ & $\rho_{3}$ & $\rho_{5}$ & $\rho_{4}$ & $\rho_{2}$ & $\rho_{4}$ & $\rho_{1}$ & 1 & $\rho_{1}$ \\
& $\mathrm{C}$ & $\rho_{5}$ & $\rho_{5}$ & $\rho_{3}$ & $\rho_{4}$ & $\rho_{4}$ & $\rho_{2}$ & $\rho_{1}$ & $\rho_{1}$ & 1 \\
\hline
\end{tabular}


Table 3. Correlation structures.

\begin{tabular}{lccccc}
\hline & $\rho_{1}$ & $\rho_{2}$ & $\rho_{3}$ & $\rho_{4}$ & $\rho_{5}$ \\
\hline Structure 1 & 0.50 & 0.00 & 0.00 & 0.00 & 0.00 \\
Structure 2 & 0.00 & 0.50 & 0.50 & 0.00 & 0.00 \\
Structure 3 & 0.00 & 0.00 & 0.00 & 0.50 & 0.50 \\
Structure 4 & 0.80 & 0.50 & 0.50 & 0.40 & 0.40 \\
Structure 5 & 0.50 & 0.70 & 0.70 & 0.30 & 0.30 \\
Structure 6 & 0.50 & 0.70 & 0.49 & 0.25 & 0.125 \\
\hline
\end{tabular}

variables collected at different waves to be weakly correlated, as disease status from the previous wave is probably not strongly related to current disease status. On the other hand, variables collected at the same wave will be correlated, as symptoms are correlated with disease status. It is easier to picture cases where variables have a similar longitudinal correlation to that of Structure 2, as this implies that data collected at the same wave are independent or weakly correlated, but a variable is moderately or strongly correlated with itself over time. We can find several variables from the data we selected from the Health and Retirement Study, a longitudinal study of US adults over 50 (Juster and Suzman 1995), similar to this structure. Additional details on the study and the selected variables can be found in Section 5. Variables like wealth, cancer diagnosis, and high blood pressure are weakly correlated with each other (correlations are between 0.024 and 0.063 ) but moderately to strongly correlated with themselves across waves (correlations range from 0.437 to 0.662 for wealth and from 0.947 to 0.995 for high blood pressure and cancer). Structure 3, where the between-wave correlation is the only nonzero correlation, is unlikely to be found in longitudinal data. All three structures were chosen because they allow us to test the performance of each design under radically different conditions.

The last three structures are meant to more accurately reflect correlations that might be seen in longitudinal data than the first three structures. We generally would not expect only one of the within-wave, between-wave, and autocorrelation to be non-zero. We also expect that the between-wave correlation would be no larger than the within-wave correlation and the autocorrelation. The correlations between wealth and income in the Health and Retirement Study are usually highest when collected at the same visit. For other variables, like diabetes, high blood pressure, heart disease, stroke, and weight, the between-wave correlations are usually similar to the within-wave correlation, which are both less than the autocorrelation. In Structure 4, within-wave correlation is largest, while for Structure 5 autocorrelation is largest. Structure 4 may be thought of as similar to Structure 1, but a variable is still correlated with itself and other variables when measured at different waves. We may imagine something like this occurring for certain diseases and symptoms, where the disease status is correlated over time, but is more strongly correlated with symptoms occurring during that visit. We can observe a correlation structure similar to Structure 5 with the variables income and wealth from the Health and Retirement Study, as the withinwave correlations range between 0.23 to 0.44 , the autocorrelations range between 0.44 and 0.66 , and the between-wave correlations range from 0.14 to 0.40 . Most variables selected from the Health and Retirement Study have a higher autocorrelation than within-wave or 
between-wave correlation. Structure 6 follows an autoregressive structure, where correlations decrease over time, with two main correlations, the correlation between different variables and the autocorrelation. For variables like high blood pressure, diabetes, cancer, heart disease, stroke, and weight in the Health and Retirement Study, we almost always see a decline in the autocorrelation when the waves are further apart. The decline is most noticeable for heart disease and stroke.

In addition, we tested the performance of each design under a random correlation structure, with the correlation computed based on a random covariance matrix. The covariance matrix is drawn from a Wishart distribution with nine degrees of freedom and a diagonal scale matrix. The Wishart distribution, a multivariate generalization of the gamma distribution, has two parameters: the degrees of freedom, which must be greater than $p-1$, and a $p \times p$ scale matrix, which must be positive definite. The scale matrix we used had ones on the main diagonal and zeros everywhere else. A diagonal matrix was chosen because the generated within-wave, between-wave, and autocorrelations would, on average, be the same and none of the correlations would be favored. After drawing a random covariance matrix, the covariance matrix was transformed into a correlation matrix. The generated correlation matrix has an expected value equal to the scale matrix used in the Wishart distribution, thus the generated correlations have means equal to zero. The degrees of freedom affect the variance of the generated correlation matrix. Specifying a large number of degrees of freedom would have resulted in very little variation in the generated correlations (i.e., all correlations would have been close to zero). We specified a small number for the degrees of freedom, generating a large range of correlations. The random correlations produced typically ranged from -0.65 and 0.65 . The use of randomly generated correlation matrices allowed us to examine which design performs the best on average across a large number of possible correlations.

We then compared performance of each design option under the proposed correlation structures by computing the variance for the variable means, variance-covariance components, and the linear change in means over time using both MLE and mutliple imputation. For maximum likelihood estimation, we calculated the variance of the means and variance-covariance components from inverting the Fisher Information using the true underlying covariance matrix. We estimated the linear change in means over time for a variable using contrasts. The variance of these linear combination of means from the contrasts can similarly be computed directly from the inverted Fisher Information matrix. For MLE, the variances of our estimates of interest did not require data simulation to compute, though we used many iterations to assess the overall performance of Option 6 , the random form assignment.

For multiple imputation, we simulated complete data from the multivariate normal distribution. Values were then set to missing so observed data matched what would have been obtained under each study design option and we performed multiple imputation. We then estimated the mean, variance- covariance, and linear change in mean over time. The mean and variance-covariance parameters were estimated from the sample mean and sample covariance matrix in each imputed data set and combined using Rubin's rules. We used a linear mixed model of the variable regressed on time for estimating the change in mean and stored the parameter estimates and standard errors for the slope and intercept. 


\subsection{Simulation Results}

The relative performance, rankings, and conclusions are largely the same when using either MLE or MI, though there is an increase in variance from the MI estimates compared to the MLE. This might be due to the finite number of imputations being used and because we assumed a known variance-covariance matrix when computing the MLE. The MI results are based on the estimated variance-covariance parameters from imputed data, which adds an additional source of variation. Since MI is more commonly used, this section will focus on simulation results using multiple imputation. The results from MLE can be found in the supplementary materials, section S1. The results in this article are based on a longitudinal data structure with three waves. For comparison, we also performed MLE for the same basic correlation structures but with two waves. The results for two waves (not shown in this article) are similar to the results for three waves.

Table 4 displays the average percent increase in variance for the mean and variancecovariance components from using the proposed split questionnaire design versus complete data. Since relative rankings for the mean and variance-covariance were very similar, we combined them into one category in the table. Similarly, Table 5 shows the variance increase for repeated measure regression for the correlation structures. All of the design options performed similarly for Structure 1, where only variables within the same wave are correlated. The longitudinal selection of split question forms did not matter very much due to the lack of correlation across waves. However, Option 5 and Option 6 did perform slightly better at estimating variable correlations due to the additional form rotations, even if most correlations were zero.

Results from Structure 2, where only the autocorrelations are non-zero, show that Option 3 performed best in terms of estimating variable means and variance-covariance

Table 4. Average percent increase in variance from complete data for mean and variance-covariance components using $M I$.

\begin{tabular}{lcrrcccr}
\hline Structure & 1 & \multicolumn{1}{c}{2} & 3 & 4 & 5 & 6 & Random \\
\hline Option 1 & 90.7 & 134.0 & 109.7 & 38.0 & 67.6 & 79.7 & 68.3 \\
Option 2 & 90.4 & 92.2 & 111.2 & 32.3 & 46.3 & 37.7 & 66.7 \\
Option 3 & 86.2 & 87.9 & 52.8 & 27.7 & 30.1 & 34.3 & 53.3 \\
Option 4 & 86.9 & 99.9 & 35.5 & 29.7 & 40.3 & 45.0 & 53.7 \\
Option 5 & 85.9 & 94.1 & 33.4 & 28.0 & 32.4 & 39.3 & 49.0 \\
Option 6 & 86.6 & 92.0 & 18.6 & 28.7 & 30.8 & 37.0 & 50.0 \\
\hline
\end{tabular}

Table 5. Average percent increase in variance from complete data for repeated measures regression change in mean over time for $M I$.

\begin{tabular}{lcrccccc}
\hline Structure & 1 & 2 & 3 & 4 & 5 & 6 & Random \\
\hline Option 1 & 51.5 & 73.0 & 89.7 & 24.1 & 42.0 & 44.6 & 44.3 \\
Option 2 & 52.9 & 102.1 & 50.5 & 41.2 & 73.5 & 61.7 & 44.7 \\
Option 3 & 52.8 & 103.0 & 31.2 & 36.3 & 50.1 & 58.9 & 37.4 \\
Option 4 & 51.9 & 82.8 & 28.4 & 30.2 & 39.3 & 45.5 & 36.5 \\
Option 5 & 51.2 & 90.7 & 23.0 & 30.5 & 36.6 & 50.2 & 33.4 \\
Option 6 & 53.9 & 94.4 & 12.2 & 33.7 & 46.6 & 57.4 & 34.4 \\
\hline
\end{tabular}


components. Option 2 and Option 6 were virtually tied for second, followed closely by Option 5. Option 1 performed the worst in estimating means and variance-covariance. The opposite occurred for estimating the change in mean over time. In this scenario, a variable is predictive of its values measured at different time points for the same subject. Since Option 2 and Option 3 rotate split questionnaire forms for all individuals, we measure each variable on a subject during the study, which enables us to better estimate missing values for that variable and leads to a better estimate for the mean and variance. On the other hand, Option 1 measures the same variables on an individual for every wave, which provides a larger sample of individuals with a variable measured at all time points than the other options, allowing a better estimate for how a variable changes over time. Option 4 and Option 5, which rotate split questionnaire forms for some individuals but not others, do not perform as well as Option 3 for estimating means and variance-covariance or as well as Option 1 for estimating the change in mean. However, the efficiency loss is not bad. They might be more useful for jointly estimating the means, variance-covariance, and change in means.

For correlation Structure 3, where a variable is only correlated with other variables measured at different waves, Option 6, the random form assignment, performed the best overall by far. Option 5 was the second best under this correlation, while Option 1 and Option 2 performed terribly. This indicates that extra form rotations and more complex designs are beneficial when between-wave correlations are large. For the random variable correlation, Option 5 and Option 6 again performed the best and Option and Option 2 did the worst. Based on this, it appears that the more complex designs perform better across all possible variable correlations; however, all correlation structures are not equally likely for longitudinal studies.

For the more realistic correlation structures (Structures 4, 5, and 6), we still see a fair amount of variability in the performance of the design options. The designs perform more similarly for correlation Structure 4, where the within-wave correlation is larger than the other correlations. Option 3 performed the best in terms of estimating the mean, variance, and covariance across correlation Structures 4, 5, and 6. Options 5 and 6 were fairly close behind. For these structures, within-wave and autocorrelation were greater than betweenwave correlation, which is likely why Option 3 outperformed Option 5 and Option 6 in estimating the sufficient statistics. Option 3 likely outperformed Option 2 because the extra rotations allowed better estimation of the between-wave correlations. Option 1 was always the worst at estimating the mean and variance-covariance, but, generally, was the best at estimating the change in mean over time; one exception being correlation Structure 5, where Option 4 and Option 5 outperform Option 1, though not by much. This indicates that there are certain instances where we might not prefer Option 1 for measuring the change in a variable over time. Option 4 and Option 5 were usually variables at each wave on a subset of study participants. Those two options are also quite a bit better at estimating the sufficient statistics compared to Option 1 and, as a result, may be preferable.

From the analysis we see that the optimal design for a longitudinal study with planned missingness depends on the structure of the data and the estimates of interest. If the variables have high autocorrelations, we prefer Option 2 or Option 3, where participants change forms every year, for estimating the mean and variance-covariance parameters, but Option 1 would be better for estimating the change in mean over time. If the data follow a 
more unusual structure, it would be better to use Option 5 or Option 6 which include more form rotations. For more realistic correlations, Option 3 appears to perform best for estimating mean and variance-covariance and Option 1 is likely best at estimating change in mean. Option 4 and Option 5 can measure both mean and variance-covariance and change in mean fairly well. While our simulations are useful for evaluating how each option performs, our data structures are more simplistic than what we would observe in practice. Next, we examine which planned missing design option works best using data from a longitudinal survey.

\section{Health and Retirement Study (HRS)}

\subsection{Overview of Selected HRS Data and Assigned Splits}

The Health and Retirement Study (HRS), beginning in 1992, is an ongoing longitudinal study of US adults age 50 and older (Juster and Suzman 1995). HRS collects information pertaining to the health, income, and job status of participants. New individuals enter the study after turning 50, while some older participants exit due to death or loss to follow up. HRS uses multistage sampling to sample households (Health and Retirement Study 2008). African Americans, Hispanics, and residents of the state of Florida are oversampled compared to the general population. Sampling weights are used to account for the unequal probability of selection. The weights include adjustments for post-stratification factors. There are numerous publications analyzing data collected from HRS. An examination of recent publications indicates analyses were frequently conducted using regression models, including linear regression (Carr et al. 2018), logistic regression (Lee et al. 2017; Pavela et al. 2018; Shah et al. 2018), Cox proportional hazards model (Shah et al. 2018), and Poisson regression (Wagner and Olson 2018). Most of the regression analyses used the sampling weights to adjust for the unequal probability of selection due to the complex sampling design. Parameter estimates and standard errors were generally reported from a weighted regression model. All of these analyses can still be performed under a split questionnaire design using multiple imputation, though, in this article we did not account for the complex sampling design.

For evaluating the performance of our proposed split questionnaire design options, we selected seven modules from the survey data collected in the 2004, 2006, 2008, and 2010 waves of the study. We primarily focused on health data from the Health and Retirement Study. The modules for diabetes and blood pressure represented Component A, the heart disease and stroke module plus the weight module represented Component $\mathrm{B}$, and the cancer module and the income and wealth module represented Component $\mathrm{C}$ of our split questionnaire design. In addition, we selected basic demographic information (age, gender, race, height, education) and past health behavior information (smoking and drinking history/status) to represent the X component measured in all participants. Several previous publications had studied the relationship between at least two of the five selected modules (diabetes, blood pressure, heart disease and stroke, weight, and income and wealth) using HRS data (Bowen 2010; Best et al. 2005; Avendano and Glymour 2008). Cancer was also selected as it is an important health condition. A total of six longitudinal modules were selected so we could divide the modules evenly into three splits. 
Each module contained multiple variables related to the main condition of interest that we selected, but, for the purpose of our analyses, we focused on only a few main variables in these modules (i.e., have you ever been diagnosed with diabetes, blood pressure, heart disease, stroke, or cancer, what is your current weight, the net value of all assets, or amount of income). The survey length (as measured by the number of questions) at each follow-up wave would have been reduced by $36.7 \%, 23.3 \%$, and $40.0 \%$ compared to the complete questionnaire for participants administered Component $\mathrm{AB}, \mathrm{AC}$, and $\mathrm{BC}$, respectively. Although AC results in the smallest reduction of survey length, AC does not include weight, potentially making it less invasive and costly compared to the other components.

The within-wave correlations, between-wave correlations, and autocorrelations differ by variable. However, in general the autocorrelations are the largest. For diabetes, blood pressure, cancer, and weight, autocorrelations were greater than 0.85. For heart disease, autocorrelations were between 0.70 and 0.90 . For stroke, autocorrelations were between 0.50 and 0.85 . Autocorrelations were weaker for income and wealth, but were still between 0.30 and 0.70 . Most within-wave correlations were between -0.30 and 0.30 , with the exception of the correlations between wealth and income, which could get as high as 0.43 . The between-wave correlations were usually very similar to within-wave correlations.

We consider all participants with no missing values in each of the seven modules for all four study waves as our complete data (ideal), a total of 3,059 subjects. The survey questions from the $\mathrm{X}$ module were taken from baseline only. We then set values to missing, mimicking the data pattern that would have been observed had we used the proposed planned missing data designs from Table 1. We also included Option 6, the random form assignment.

\subsection{Analysis and Results from HRS Data}

We performed multiple imputation on the missing values using MICE (Van Buuren and Groothuis- Oudshoorn 2011). The same imputation model was used for each option. Linear regression models were used to impute normally distributed variables and logistic regression models were used to impute binary variables. A variable was imputed conditional on itself at previous waves along with other health conditions and demographic data. A variable was not necessarily imputed conditional on all selected variables due to issues with collinearity for all regression models and separation of data points in logistic regression. However, care was taken to make sure the imputation model was consistent with the model later used to analyze the variables. If a variable was transformed in the later analysis, then it was transformed in the imputation. Only the main variables from each module that were used in our analyses were included in the imputation models.

To assess the validity of the imputations, we examined the marginal distribution of the imputed values compared to the observed values and checked for any major discrepancies between the two. Since data are MCAR by design, we would not expect the marginal distribution of imputed values to differ from the observed values. Furthermore, we performed diagnostics on the regression models through goodness of fit testing and diagnostic plots to examine the validity of our imputation models. For linear regression models, we plotted the residuals versus the fitted values, shown in Figure 1. If the imputation model was correctly specified then we would expect to see a random scatter 


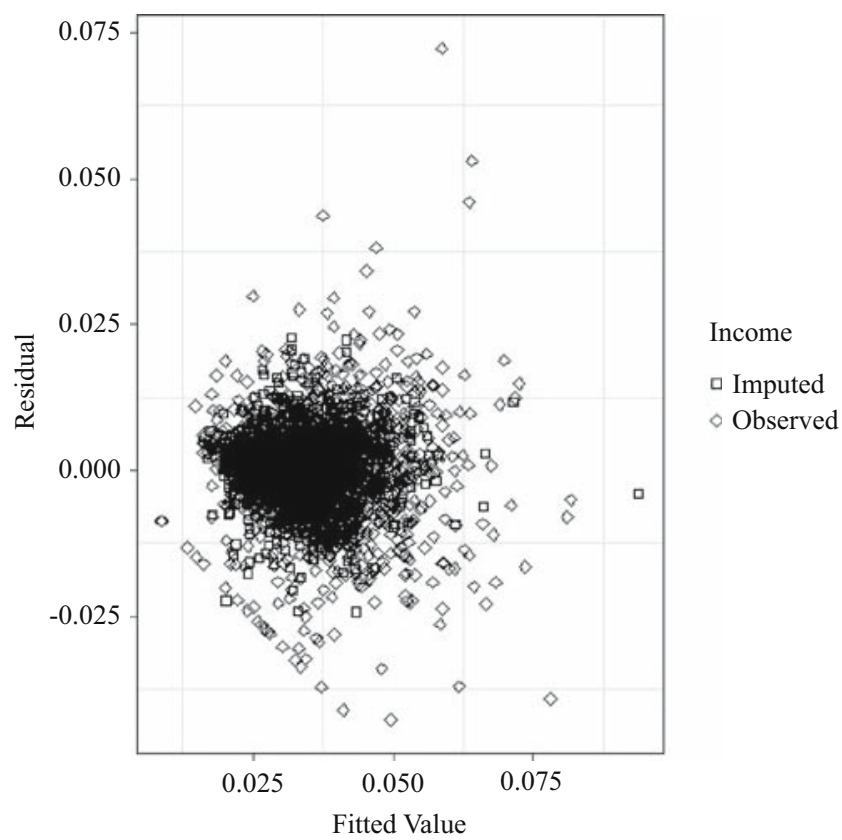

Fig. 1. Imputation diagnostic plot of residuals versus fitted values for transformation of 2010 income.

centered around the X-axis, with no discernible difference between observed and imputed values, like in Figure 1.

After performing multiple imputation, we analyzed estimates for the marginal distributions of the variables by examining the estimated mean, median, and quartiles of the continuous variables under the different options. For categorical variables we estimated $\pi_{j}$, the probability that variable $j$ is equal to one. For each population parameter estimate, we examined the bias under each proposed design by taking the difference between the estimates under the planned missing designs and the estimates from the complete data. The differences for the mean and quantile estimates were standardized by dividing by the complete data standard deviation of the variables, while the difference in $\pi_{j}$ was standardized by dividing by the square root of $\pi_{j}\left(1-\pi_{j}\right)$ under complete data. Figure 2 plots the distribution of the standardized differences under the six options. Option 3 produced the best estimates for univariate parameters with the bias distributed tightly around zero, with Option 2 coming in a close second. The other options performed similarly, though Option 1 performed slightly worse. In addition to examining the bias of estimated parameters, we also examined the performance of each design based on how the multiple imputation variance of the estimated population mean under each split questionnaire design compared to the variance with complete data. Figure 3 displays the ratio of the standard error of the mean estimate for each option to the standard error from the complete data. The ratio of the standard error demonstrates how much larger the standard error for the split questionnaire design is compared to the complete data. This is similar to the idea of estimating the fraction of missing information for multiple imputation (Little and Rubin 2002 chap. 10, 211), which gives an estimate for how much 


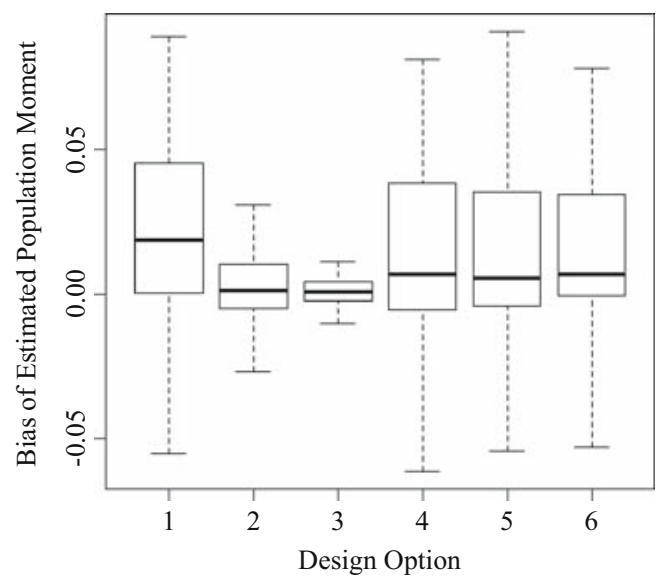

Fig. 2. Distribution of standardized bias for univariate estimates.

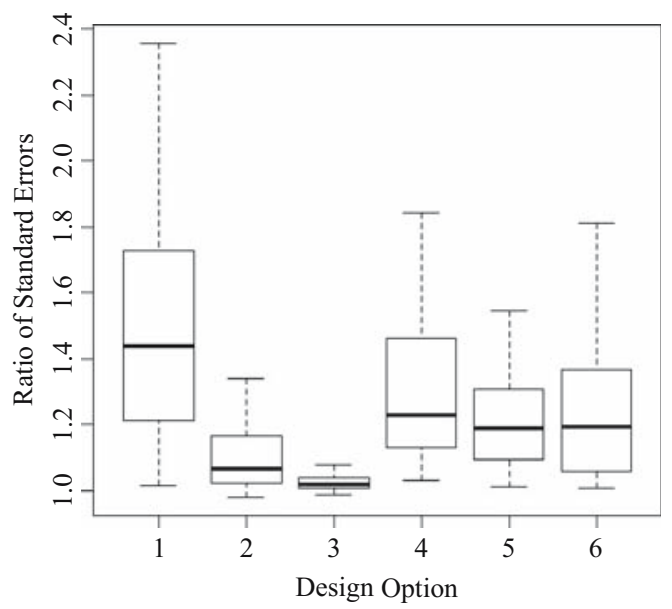

Fig. 3. Ratio of the standard error of the estimated mean to complete data standard error.

the variance of an estimate has increased due to missing data. In this instance, we do not need to estimate the fraction of missing information because we can directly determine the loss of efficiency. Once again, Option 3 performed best, generally producing smaller standard errors, followed by Option 2, while Option 1 performed the worst.

We also evaluated the performance of each option using three different regression analyses based on three previous publications with HRS data, (Bowen 2010; Best et al. 2005; Avendano and Glymour 2008). More details on the regression models and results based on these papers can be found in the supplementary materials, Section S2.

Figure 4 shows how each option performed in terms of parameter estimation bias across the three regression models, by taking the difference in parameter estimates under the design option and the complete data and dividing by the complete data standard error. Option 3 performed the best at estimating regression coefficients. Option 2 was second 


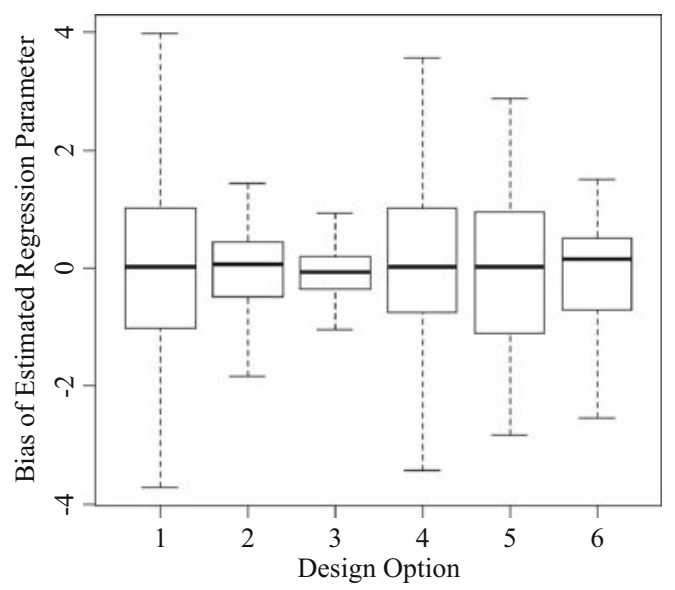

Fig. 4. Distribution of standardized regression parameter estimation bias.

best, followed by Option 6. The other three options performed similarly overall in terms of bias, though Option 1 was generally worse. In Figure 5, we display the ratio of the MI standard errors under each option to the complete data standard errors. From the figure, we observe that Option 3 produced the smallest standard errors. The other options performed fairly similar to each other, though the average standard error is slightly smaller for Option 2 and Option 6 compared to the other options.

Option 3 easily performed the best overall for the HRS data. Option 2 was the second best, while Option 1 performed the worst. The strong performance of Option 2 and 3 in this instance is not surprising since the autocorrelation was quite large for most variables, and we saw from simulations that Option 2 and Option 3 performed better in terms of mean and variance estimation when the autocorrelation is much greater than the other correlations. Had the within-wave and between-wave correlations been stronger, Option 5 and Option 6 would likely have performed better.

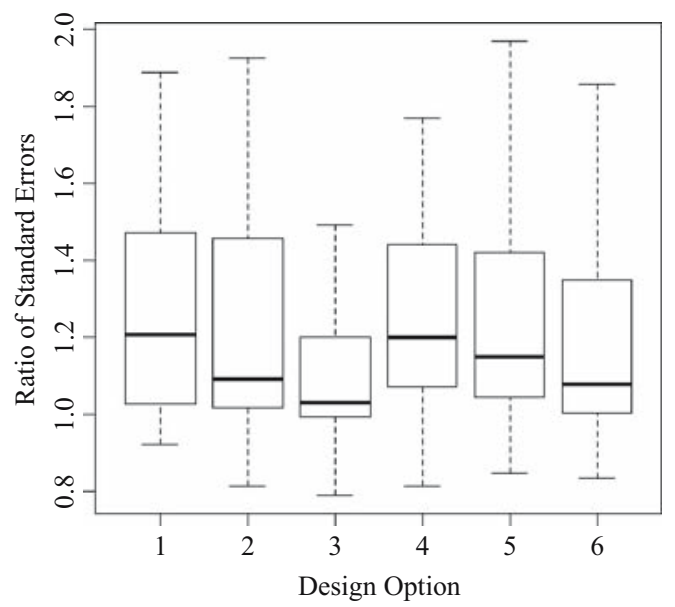

Fig. 5. Distribution of the ratio of regression parameter standard error to complete data standard error. 


\section{Discussion}

\subsection{Conclusions}

The use of planned missing data in a survey study can reduce the burden and fatigue on participants, leading to an increase in the quality of the data and a reduction in unplanned missing values. This could also reduce the dropout rate in longitudinal studies. There is a good deal of literature on implementing planned missing designs in cross-sectional studies, commonly using a three-form design, but until recently there has been little research on implementing planned missing data designs in longitudinal studies, though Jorgensen et al. (2014) did consider multiple assignment methods for three-form survey designs of longitudinal studies in a latent variable setting.

In our article, we examined six different design options for allocating forms in a longitudinal study. We observed from our simulations and MLE analysis that the performance of each design option depended on the correlation structure of the data and which estimands were of interest. We also tested these designs on survey data from the HRS. To truly determine which design is the best, we need to understand the longitudinal correlation structure of the data. We could wait until we have at least two waves of data before applying a planned missing data design to the longitudinal study. However, the optimal design mostly depends on the between-wave correlation, within-wave correlation, and autocorrelation. We can estimate the within-wave correlations from the first wave before applying a longitudinal design. The between-wave correlations are unlikely to be larger than the within-wave correlations, based on what we observed in the HRS data. Thus, we only need to judge whether the autocorrelation is larger than the within-wave correlation and which estimands are of interest to determine which design to apply.

If investigators are primarily concerned with estimating the mean, regression of one variable on a subset of variables, or cross-sectional properties of the data, we recommend implementing Option 3. Option 3 always outperformed the best for most longitudinal studies due to large autocorrelations. Option always outperformed the simpler Option 2, even though both designs similarly administered a different form to a participant at every visit. Sometimes, Option 3 did considerably better than Option 2. The only reason to use Option 2 over Option 3 is that it requires fewer group assignments, making it easier to implement. Option 3 performed the best, or close to it, in terms of estimating the means and variance- covariance for every correlation structure except for Structure 3, where between-wave correlations were the only non-zero correlation, and the random correlation structure. However, the autocorrelation and within-wave correlations are likely to be stronger than between-wave correlations for longitudinal data. In these situations Option 3 was the best.

The more complex design options (Options 3, 4 and 5) generally outperformed the simpler options (Options 1 and 2) in terms of estimating the means and variancecovariance in our simulations, except for correlation Structures 2 and 6, where Option 2 outperformed Option 4 and Option 5. In these instances, the autocorrelation was quite strong compared to the between-wave correlation. The autocorrelations were quite large for the HRS data as well, which may be the reason why Option 2 performed better; however, Option 3 was still better than Option 2. 
If investigators are only interested in the change in a variable over time, which may often be the case for longitudinal studies, Option 1 will usually be the best design option. This is a direct result of measuring the same variables on a participant at each time point. This result is consistent with results from Jorgensen et al. (2014), which found that the same form produces more precise estimates for autoregressive parameters. Correlation Structures 3 and 5 and the random correlation structure were the only instances where Option 1 did not perform the best or very close to the best in terms of estimating the change over time, though it was not that far behind the best option for Structure 5. Structure 5 had moderately strong with-wave and between-wave correlations and strong autocorrelations. The fairly strong overall correlations may have helped the more complex options outperform Option 1 in this instance.

Even if the change in variables over time is of primary interest, we still recommend considering Option 4 and Option 5. Option 1 was generally the worst design in terms of estimating the mean, variance, and covariance. Additionally, Option 1 did not perform well for the HRS data. Option 4 generally performed similarly to Option 1 in terms of estimating change in mean over time, but performed better in estimating the mean and variance-covariance. This may be a good design to use if interest lies in estimating the mean and variance-covariance, in addition to the change in mean. The ratio of participants receiving the same form and receiving different forms could also be altered for Option 4 and Option 5, depending on which estimand is of greater interest. Seeing how Option 3 always outperformed Option 2, redesigning Option 5 so that half of the participants received the same form would likely perform similar to Option 4 in terms of estimating the change over time, but would be better for estimating the mean and variance-covariance. Depending on the strength of the overall correlations, Option 4 and Option 5 may be better than Option 1 in terms of estimating the change in mean over time. Even though the random form assignment performed fairly well in most instances, we would recommend using Option 5 instead, as it allows us to measure the same variables every year in a subset of participants. Measuring the same set of variables in at least a subset of participants is useful for estimating the change in a variable over time.

It is also important to keep in mind that when selecting design options, certain higherorder interactions may not be directly estimable, which is also an issue for three-form split questionnaire designs in cross-sectional studies. For example, research suggests that how much influence a person believes they have over events in their lives and a person's control over his or her work environment may modify the effect of stress on health (Meier et al. 2008). If it is of interest to investigate a higher order interaction, such as the example above, care must be taken when designing the split questionnaire. Although all two-way interactions can be estimated using the three-form design, not all three-way or higher-order interactions can be estimated. Any interaction that includes a variable in component $\mathrm{A}$, a variable in component $\mathrm{B}$, and a variable in component $\mathrm{C}$ is not directly estimable. We also have to consider that some interactions involving components measured at different time points might not be estimable with the longitudinal designs. For example, Option 2 and Option 3 do not allow a three-way interaction of a variable measured in the same component across all three waves, since that variable is never measured in the same individuals in all three waves. Options 1, 4 and 5 do not have this problem. It should be taken into account during the design stage if certain higher-order interactions are of importance. 


\subsection{Limitations and Future Research}

We evaluated the proposed design options using simulations and HRS data. Although the HRS data contained both binary and continuous variables, we did not examine any joint distributions of variables other than multivariate normal for our simulations. It would be useful to examine whether the conclusions from our simulations are affected by different variable distributions. We might want to consider instances where we have binary, categorical, or count variables in addition to the continuous multivariate normal distribution. Although, in principle, we would not expect a huge difference from the multivariate normal case (as we saw with the results from the HRS data), the distribution of our variables affects the imputation models and could result in different conclusions.

Prior information regarding the data structure would help in determining the optimal design option for a study and can help in the imputation. Several papers have considered dividing the survey questions into forms based on correlation structures or other methods to improve estimation, but require prior information on the variables (Raghunathan and Grizzle 1995; Thomas et al. 2006; Adigiizel and Wedel 2008). These methods were not considered when constructing our cross-sectional split questionnaire forms. Using these methods could potentially affect which of our longitudinal design options performs best. The results of the longitudinal split questionnaire designs were affected by cross-sectional item assignment in Jorgensen et al. (2014). We should also be aware that our results when using planned missing designs will be biased if we do not specify a correct imputation model, which by no means is a trivial matter. Even when data are MCAR, there are potential issues with collinearity, separation of data points, and violations of parametric distributions for conditional regression imputation models.

Our proposed longitudinal split questionnaire designs use the same basic three-form split questionnaire throughout the study. We could create new split questionnaire forms at every visit based on the observed data from previous visits. We could consider adaptive allocations of forms, where a participant's form at the next visit is based on previous responses. The large number of potential split questionnaires to consider at every visit makes this approach more complicated. Our proposed designs are much easier to implement. It would be a good idea to examine how the efficiency of our proposed designs compares to this approach.

One further limitation is that we did not consider the effect that complex survey designs might have on our proposed methods. Simple random sampling was implicitly assumed for our analyses; however, most large-scale studies use complex survey designs, such as multistage sampling (Zhou et al. 2016). For stratified and/or clustered sampling, our results and conclusions for administering split questionnaire designs should hold within each strata and/or primary sampling units (PSUs). For the multivariate normal distribution, overall estimates for the mean and regression parameters could be obtained by taking a weighted average of the parameter estimates within each group defined by the strata and/or PSUs, assuming we have enough observations within each group (Dumouchel and Duncan 1983). It would likely be preferable to assign split questionnaires within groups and, in some circumstances, it might be useful to apply different longitudinal designs in different groups.

When there is a small number of observations per group or our study involves poststratification, we might use survey weights instead of computing parameters within 
groups. In the multivariate normal case, weighted least squares could be used for estimating regression parameters (Dumouchel and Duncan 1983). Means could be computed as a weighted average. Both the means and regression parameters can be obtained by applying transformations to the data using the weights. Our conclusions for longitudinal split questionnaire designs would likely still hold, provided the transformations do not alter the relative strengths of the within-wave and between-wave correlations and the autocorrelations from the unweighted analyses. A more thorough investigation of the effect of complex survey designs on longitudinal split questionnaire designs should be performed.

We note that though our proposed methods for longitudinal split questionnaire designs differ from rotating panel surveys and the wave missing design described in Little and Rhemtulla (2013), they are motivated by a similar concern of reducing respondent burden in longitudinal studies. In rotating panel surveys, participants are only enrolled in a panel survey for a finite period of time and new participants are enrolled in the study at each wave to replace the exiting participants. In wave missing designs, not all participants are interviewed at every wave of the study. Both designs reduce the interview burden on participants. Each design could be used in conjunction with our split questionnaire design methods to further reduce burden for longitudinal studies.

\section{References}

Adams, L.L.M., and G. Darwin. 1982. "Solving the Quandary Between Questionnaire Length and Response Rate in Educational Research." Research in Higher Education 17(3): 231-240. DOI: http://dx.doi.org/10.1007/BF00976700.

Adigüzel, F., and M. Wedel. 2008. "Split Questionnaire Design for Massive Surveys." Journal of Marketing Research 45(5): 608-617. DOI: https://dx.doi.Org/10.1509/jmkr. 45.5.608.

Avendano, M., and M.M. Glymour. 2008. "Stroke Disparities in Older Americans: Is Wealth a More Powerful Indicator of Risk than Income and Education?" Stroke 39(5): 1533-1540. DOI: https://dx.doi.org/10.1161/STRCIKEAHA.107.490383.

Best, L.E., M.D. Hayward, and M.M. Hidajat. 2005. "Life Course Pathways to AdultOnset Diabetes." Social Biology 52(3-4): 94-111. DOI: https://dx.doi.org/10.1080/ 19485565.2005.9989104.

Bowen, M.E. 2010. "Coronary Heart Disease from a Life-Course Approach: Findings from the Health and Retirement Study, 1998-2004." Journal of Aging and Health 22(2): 219-241. DOI: https://dx.doi.org/10.1177/0898264309355981.

Box, G.E.P., and D.A. Pierce. 1970. "Distribution of Residual Autocorrelations in Autoregressive- Integrated Moving Average Time Series Models.” Journal of the American Statistical Association 65(332): 1509-1526. DOI: https://dx.doi.org/10.2307/2284333.

Carr, D.C., S. Ureña, and M.G. Taylor. 2018. “Adjustment to Widowhood and Loneliness Among Older Men: The Influence of Military Service." Gerontologist 58(6): 1085-1095. DOI: doi.org/10.1093/geront/gnx110.

Chhabra, G., V. Vashish, and J. Ranjan. 2017. "A Comparison of Multiple Imputation Methods for Data with Missing Values." Indian Journal of Science and Technology 10(19). DOI: https:/dx.doi.org/10.17485/ijst/2017/v10i19/110646. 
Childs, R.A. and A.P. Jaciw. 2002. "Matrix Sampling of Items in Large-Scale Assessments." Practical Assessment, Research and Evaluation 8(16). DOI: https://dx. doi.org/10.7275/gwvh-4z51.

Chipperfield, J.O., M.L. Barr, and D.G. Steel. 2018. "Split Questionnaire Designs: Collecting Only the Data that You Need through MCAR and MAR Designs." Journal of Applied Statistics 45(8): 1465-1475. DOI: https://dx.doi.org/10.1080/02664763.2017. 1375085.

Chipperfield, J.O., and D.G. Steel. 2009. "Design and Estimation for Split Questionnaire Surveys.” Journal of Official Statistics 25(2): 227-244. DOI: https://dx.doi.org/10.1.1. 894.1568\&rep $=$ rep1\&type $=$ pdf.

Chipperfield, J.O., and D.G. Steel. 2011. "Efficiency of Split Questionnaire Surveys." Journal of Statistical Planning and Inference 141(5): 1925-1932. DOI: https://dx. doi.org/10.1016Zj.jspi.2010. 12.003.

Cochran, W.G. 1977. Sampling Techniques. New York: John Wiley \& Sons, Inc. 3rd ed. Collins, L.M., J.L. Schafer, and C.M. Kam. 2001. "A Comparison of Inclusive and Restrictive Strategies in Modern Missing Data Procedures.” Psychological Methods 6(4): 330-351. DOI: http://dx.doi.org/10.1037/1082-989X.6.4.330.

Cook, N.R., and J.H. Ware. 1983. "Design and Analysis Methods for Longitudinal Research." Annual Review of Public Health 4: 1-23. DOI: https://dx.doi.org/10.1146/annurev.pu.04.050183.000245.

Creech, B., M. Smith, J. Davis, L. Tan, N. To, S. Fricker, and J.M. Gonzalez. 2011. Measurement Issues Study Final Report. BLS Internal Report. Available at: https://www.bls.gov/cex/research_papers/pdf/cesrvmeth_davis.pdf (accessed March 2019).

Deutskens, E., A. Jong, K. de Ruyter, and M. Wetzels. 2006. "Comparing the Generalizability of Online and Mail Surveys in Cross-National Service Quality Research." Marketing Letters 17: 119-136. DOI: https://dx.doi.org/10.1007/s11002006-4950-8.

Dillman, D., M.D. Sinclair, and J.R. Clark. 1993. "Effects of Questionnaire Length, Respondent- Friendly Design, and a Difficult Question on Response Rates for Occupant-Addressed Census Mail Surveys." Public Opinion Quarterly 57(3): 289-304. DOI: https://dx.doi.org/10.1086/269376.

Dumouchel, W.H., and G.J. Duncan. 1983. "Using Sample Survey Weights in Multiple Regression Analyses of Stratified Samples." Journal of the American Statistical Association 78(383): 535-543. DOI: https://dx.doi.org/10.1080/01621459.1983. 10478006.

Galesic, M., and M. Bosnjak. 2009. "Effects of Questionnaire Length on Participation and Indicators of Response Quality in a Web Survey." Public Opinion Quarterly 73(2): 349-360. DOI: http://10.1093/poq/nfp031.

Gonzalez, J.M. 2012. The Use of Responsive Split Questionnaires in a Panel Survey. PhD diss. University of Maryland. Available at: https://drum.lib.umd.edu/handle/1903/13171 (accessed March 2019).

Gonzalez, J.M., and J.L. Eltinge. 2007. "Multiple Matrix Sampling: A Review." In Proceedings of the Section on Survey Research Methods: American Statistical Association, July 29, 2007, 3069-3075. Alexandria, VA: American Statistical 
Association. Available at: http://www.amstat.org/sections/srms/Proceedings/y2007/ Files/JSM2007-000494.pdf (accessed October 2015).

Gonzalez, J.M., and J.L. Eltinge. 2008. "Adaptive Matrix Sampling for the Consumer Expenditure Quarterly Interview Survey.” In Proceedings of the Section on Survey Research Methods, American Statistical Association, August 3-7, 2008, 2081-2088. Alexandria, VA: American Statistical Association. Available at: http://www.asasrms. org/Proceedings/y2008/Files/301351.pdf (accessed March 2017).

Graham, J.W., B.J. Taylor, A.E. Olchowski, and P.E. Cumsille. 2006. "Planned Missing Data Designs in Psychological Research." Psychological Methods 11(4): 323-343. DOI: https://dx.doi.org/10.1037/1082-989X.11.4.323.

Groves, R.M. 1989. Survey Errors and Survey Costs. Hoboken, New Jersey: John Wiley \& Sons, Inc.

Hardt, J., M. Herke, and R. Leonhart. 2012. “Auxiliary Variables in Multiple Imputation in Regression with Missing X: A Warning Against Including too many in Small Sample Research.” BMC Medical Research Methodology 12(1): 184. DOI: https://dx.doi.org/ 10.1186/1471-2288-12-184.

Hartley, H., and R. Hocking. 1971. "The Analysis of Incomplete Data." Biometrics 27(4): 783-823. DOI: https://dx.doi.org/10.2307/2528820.

Health and Retirement Study. 2008. Sample Evolution: 1992-1998. Ann Arbor, MI: Institute for Social Research, University of Michigan. Available at: http://hrsonline.isr. umich.edu/sitedocs/surveydesign.pdf (accessed March 2019).

Herzog, A.R., and J.G. Bachman. 1981. "Effects of Questionnaire Length on Response Quality." Public Opinion Quarterly 45(4): 549-559. DOI: https://dx.doi.org/10.1086/ 268687.

Jorgensen, T.D., M. Rhemtulla, A. Schoemann, B. McPherson, W. Wu, and T.D. Little. 2014. "Optimal Assignment Methods in Three-Form Planned Missing Data Designs for Longitudinal Panel Studies.” International Journal of Behavioral Development 38(5): 397-410. DOI: https://dx.doi.org/10.1177/0165025414531094.

Juster, F.T., and R. Suzman. 1995. "An Overview of the Health and Retirement Study." Journal of Human Resources 30: S7-S56. DOI: https://dx.doi.org/10.2307/146277.

Kaplan, D., and D. Su. 2016. "On Matrix Sampling and Imputation of Context Questionnaires with Implications for the Generation of Plausible Values in Large-Scale Assessments." Journal of Educational and Behavioral Statistics 41(1): 57-80. DOI: https://dx.doi.org/10.3102/1076998615622221.

Kenward, M.G., and G. Molenberghs. 1998. "Likelihood Based Frequentist Inference When Data Are Missing at Random.” Statistical Science 13(3): 236-247. DOI: https:// dx.doi.org/10.1214/ss/1028905886.

Lee, M., M.M. Khan, and B. Wright. 2017. "Is Childhood Socioeconomic Status Related to Coronary Heart Disease? Evidence from the Health and Retirement Study (1992-2012)." Gerontology \& Geriatric Medicine 3: 1-9. DOI: https://dx.doi.org/10. $1177 / 2333721417696673$.

Little, T.D., and M. Rhemtulla. 2013. "Planned Missing Data Designs for Developmental Researchers." Child Development Perspectives 7(4): 199-204. DOI: https://dx.doi.org/ 10.1111/cdep. 12043. 
Little, R.J.A., and D.B. Rubin. 2002. Statistical Analysis with Missing Data. Hoboken, New Jersey: John Wiley \& Sons, Inc. 2nd ed.

Littvay, L. 2009. "Questionnaire Design Considerations with Planned Missing Data." Review of Psychology 16(2): 103-113.

Meier, L.L., N.K. Semmer, A. Elfering., and N. Jacobshagen. 2008. "The Double Meaning of Control: Three-Way Interactions Between Internal Resources, Job Control, and Stressors at Work." Journal of Occupational Health Psychology 13(3): 244-258. DOI: https://dx.doi.org/10.1037/1076-8998. 13.3.244.

Pavela, G., Y.I. Kim, and S.J. Salvy. 2018. "Additive Effects of Obesity and Loneliness on C-reactive Protein.” PLOS One 13(11): e0206092. DOI: https://dx.doi.org/10.1371/journal.pone.0206092.

Peytchev, A. and E. Peytcheva. 2017. "Reduction of Measurement Error Due to Survey Length: Evaluation of the Split Questionnaire Design Approach.” Survey Research Methods 11(4): 361-368. DOI: http://dx.doi.org/10.18148/srm/2017.v11i4.7145.

Raghunathan, T.E. and J.E. Grizzle. 1995. "A Split Questionnaire Survey Design." Journal of the American Statistical Association Statistical Association 90(429): 54-63. DOI: https://dx.doi.org/10.2307/2291129.

Raghunathan, T.E., J.M. Lepkowski, J. van Hoewyk, and P. Solenberg. 2001. “A Multivariate Technique for Multiply Imputing Missing Values Using a Sequence of Regression Models.” Survey Methodology 27(1): 85-95.

Raghunathan, T.E., P.W. Solenberger, and J. van Hoewyk. 2002. IVEware: Imputation and Variance Estimation Software User Guide. Ann Arbor, MI: Institute for Social Research, University of Michigan. Avalilable at: ftp.isr.umich.edu/pub/src/smp/ive/ive_user.pdf (accessed September 2014).

Rhemtulla, M., and T. Little. 2012. "Tools of the Trade: Planned Missing Data Designs for Research in Cognitive Development." Journal of Cognition and Development: Official Journal of the Cognitive Development Society 13(4). DOI: https://dx.doi.org/ 10.1080/ 15248372.2012.717340.

Roszkowski, M.J., and A.G. Bean. 1990. "Believe it or not! Longer Questionnaires have Lower Response Rates.” Journal of Business and Psychology 4(4): 495-509. DOI: https://dx.doi.org/10.1007/BF01013611.

Rubin, D.B. 1976. "Inference and Missing Data." Biometrika 63(3): 581-592. DOI: https:// dx.doi.org/10.2307/2335739.

Rubin, D.B. 1987. Multiple Imputation for Nonresponse in Surveys. New York: John Wiley \& Sons, Inc.

Schuman, H., and S. Presser. 1981. Questions and Answers in Attitude Surveys: Experiments on Question Form, Wording, and Context. New York: Academic Press.

Shah, M., D. Paulson, and V. Nguyen. 2018. "Alcohol Use and Frailty Risk among Older Adults over 12 Years: The Health and Retirement Study." Clinical Gerontologist 41(4): 315-325. DOI: https://dx.doi.org/10.1080/07317115.2017.1364681.

Sharp, L.M., and J. Frankel. 1983. "Respondent Burden: A Test of Some Common Assumptions." Public Opinion Quarterly 47(1): 36-53. DOI: https://dx.doi.org/10. 1086/268765.

Shoemaker, D.M. 1973. Principles and Procedures of Multiple Matrix Sampling. Cambridge, MA: Ballinger Publishing Company. 
Sudman, S., N.M. Bradburn, and N. Schwarz. 1996. Thinking about Answers: The Application of Cognitive Processes to Survey Methodology. San Francisco: JosseyBass.

Thomas, N., T.E. Raghunathan, N. Schenker, M.J. Katzoff, and C.L. Johnson. 2006. “An Evaluation of Matrix Sampling Methods Using Data from the National Health and Nutrition Examination Survey." Survey Methodology 32(2): 217-231.

Van Buuren, S., and K. Groothuis-Oudshoorn. 2011. "MICE: Multivariate Imputation by Chained Equations in R." Journal of Statistical Software 45(3): 1-67. Doi https://dx. doi.org/10.18637/jss.v045.i03.

Wagner, J., and K. Olson. 2018. "An Analysis of Interviewer Travel and Field Outcomes in Two Field Surveys.” Journal of Official Statistics 34: 211-237. DOI: https://dx.doi. org/10.1515/jos-2018-0010.

Yansaneh, I.S. 2005. "An Analysis of Cost Issues for Surveys in Developing and Transition Countries." In Household Sample Surveys in Developing and Transition Countries. 253-266. New York: United Nations. Available at: https://unstats.un.org/unsd/hhsurveys/pdf/Household_surveys.pdf (accessed November 2019).

Zabel, J.E. 1998. "An Analysis of Attrition in the Panel Study of Income Dynamics and the Survey of Income and Program Participation with an Application to a Model of Labor Market Behavior." The Journal of Human Resources 33(2): 479-506. DOI: https://dx. doi.org/10.2307/146438.

Zhou, H., M.R. Elliott, and T.E. Raghunathan. 2016. "Synthetic Multiple-Imputation Procedure for Multistage Complex Samples.” J Off Stat 32(1): 231-256. DOI: https:// dx.doi.org/10.1515/JOS-2016-0011.

Received October 2018

Revised March 2019

Accepted April 2020 